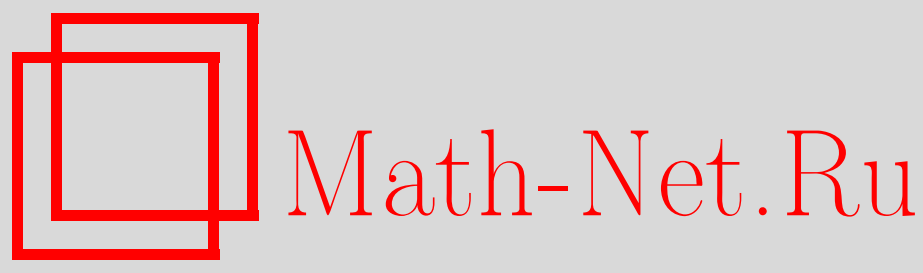

М. А. Соловьев, Скрученная свертка и звездочное произведение Мойала обобщенных функций, ТМФ, 2012, том 172, номер 1, 9-27

DOI: https://doi.org/10.4213/tmf6939

Использование Общероссийского математического портала Math-Net.Ru подразумевает, что вы прочитали и согласны с пользовательским соглашением http://www . mathnet.ru/rus/agreement

Параметры загрузки:

IP : 54.197 .130 .99

26 апреля 2023 г., 16:48:53

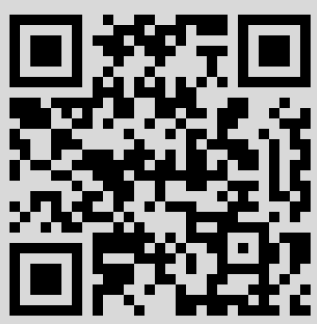




\title{
СКРУЧЕННАЯ СВЕРТКА И ЗВЕЗДОЧНОЕ ПРОИЗВЕДЕНИЕ МОЙАЛА ОБОБЩЕННЫХ ФУНКЦИЙ
}

\begin{abstract}
Рассматриваются ядерные пространства функций, на которых задано непрерывное действие группы Вейля-Гейзенберга, и исследуются основные свойства скрученной свертки функций с элементами сопряженного пространства. Итоговая теорема характеризует соответствующую алгебру свертывателей и показывает, что она содержит все достаточно быстро убывающие функционалы из сопряженного пространства. Как следствие получено общее описание алгебры мойаловских мультипликаторов фурье-преобразованного пространства. Результаты распространяют исчисление вейлевских символов за традиционные рамки распределений умеренного роста.
\end{abstract}

Ключевые слова: произведение Мойала, скрученная свертка, вейлевские символы, группа Вейля-Гейзенберга, некоммутативная теория поля, топологические *-алгебры, пространства обобщенных функций.

\section{1. ВВЕДЕНИЕ}

Скрученная свертка функций $g_{1}(s)$ и $g_{2}(s)$ на линейном симплектическом пространстве является некоммутативной деформацией обычной свертки и определяется формулой

$$
\left(g_{1} \circledast_{\theta} g_{2}\right)(s)=\int g_{1}(t) g_{2}(s-t) e^{i \theta(s, t) / 2} d t,
$$

где $\theta$ - билинейная кососимметрическая форма, задающая симплектическую структуру $^{1)}$. Преобразование Фурье переводит скрученную свертку в звездочное произведение $\star_{\theta}$ Вейля-Греневольда-Мойала, которое выражает закон композиции вейлевских символов операторов квантовой механики и играет ключевую роль в вейлевском квантовании (см. [1], [2]). Стоит отметить, что первоначально закон композиции функций на фазовом пространстве, соответствующий произведению операторов в гильбертовом пространстве, был записан фон Нейманом [3] именно в терминах скрученной свертки. Обычно звездочное произведение и скрученную свертку исходно определяют для гладких и быстро убывающих функций, составляющих

\footnotetext{
1) Скрученную свертку часто обозначают символом $*_{\theta}$, но в настоящей статье будет использоваться символ $\circledast_{\theta}$ во избежание путаницы со звездочным произведением $\star_{\theta}$.
}

*Физический институт им. П. Н. Лебедева РАН, Москва, Россия. E-mail: soloviev@lpi.ru 
пространство Шварца $S$, которое является ассоциативной топологической алгеброй относительно каждой из этих операций, но на практике приходится иметь дело с их продолжением на то или иное (в зависимости от решаемой задачи) подпространство в сопряженном пространстве $S^{\prime}$ распределений умеренного роста. Максимальное расширение по дуальности было предложено Антонецем [4]-[6] и состоит в построении алгебры мультипликаторов для алгебры $\left(S, \star_{\theta}\right)$, или, что равносильно, для алгебры $(S, \circledast \theta)$. Затем оно изучалось во многих работах, наиболее глубоко в [7]-[9] (подробный обзор и ссылки имеются в [10]).

В последние 15 лет интенсивно исследуются модели теории поля на некоммутативных пространствах, основанные на использовании мойаловского звездочного произведения (см. обзор [11] для введения в эту тематику). Интерес к некоммутативным пространствам был стимулирован анализом [12], [13] ограничений на точность локализации пространственно-временных событий в квантовой теории, включающей гравитацию, а также изучением низкоэнергетического предела теории струн [14]. Есть основания считать [15]-[17], что рамки распределений умеренного роста слишком узки для последовательной формулировки общих принципов некоммутативной квантовой теории поля. Мойаловское произведение нелокально, и представляющее его разложение по параметру некоммутативности $\theta$ сходится лишь на аналитических функциях, фурье-образы которых убывают быстрее, чем гауссова экспонента [18], [19]. Анализ нарушений микропричинности в простейших некоммутативных моделях [20]-[22] указывает на возможную связь некоммутативной теории поля с рассматривавшимися ранее нелокальными теориями, где квантовые поля трактуются как операторнозначные обобщенные функции над подходящим пространством аналитических пробных функций вместо пространства Шварца. Возникает задача соответствующего обобщения исчисления вейлевских символов.

В работе [18] установлено условие, при котором ядерное пространство пробных функций $E\left(\mathbb{R}^{d}\right)$, имеющее структуру топологической алгебры относительно обычной свертки, является алгеброй и относительно скрученной свертки, а фурье-двойственное ему пространство является алгеброй относительно произведения Мойала. Это условие можно записать как

$$
e^{-i \theta / 2} \in M(E \widetilde{\otimes} E)
$$

где $E \widetilde{\otimes} E$ - пополненное проективное тензорное произведение, отождествимое с $E\left(\mathbb{R}^{2 d}\right)$ в силу теоремы о ядре, и $M(E \widetilde{\otimes} E)$ - пространство его мультипликаторов относительно обычного поточечного произведения. В случае пространств $S_{\beta}^{\alpha}$ Гельфанда-Шилова [23], рассмотренном в работе [18], условие (2) приводит к ограничению $\alpha \geqslant \beta$ на задающие пространство индексы. Точное описание поточечных мультипликаторов для пространств $S_{\beta}^{\alpha}$ дано Паламодовым [24], а соответствующие им алгебры мойаловских мультипликаторов построены в [25].

Здесь мы покажем, что данная задача может быть решена в общем виде и построение алгебры мойаловских мультипликаторов, включая описание ее элементов, можно выполнить для любого полного ядерного бочечного ${ }^{2)}$ пространства, на котором непрерывно действует группа Вейля-Гейзенберга, причем это удается сделать без сложных аналитических выкладок, используя лишь хорошо известные факты [26]

2)Этими свойствами обладают практически все пространства, используемые в теории обобщенных функций; см. [26] относительно их определения и роли в функциональном анализе. 
о тензорных произведениях ядерных пространств. Основное наблюдение состоит в том, что из условия (2) следует

$$
v \circledast_{\theta} g \in M(E), \quad g \circledast_{\theta} v \in M(E) \quad \text { при всех } g \in E, v \in E^{\prime},
$$

где $M(E)$ - пространство поточечных мультипликаторов для $E$. Это позволяет достаточно точно охарактеризовать те элементы сопряженного пространства $E^{\prime}$, которые являются мультипликаторами алгебры $\left(E, \circledast_{\theta}\right)$.

Статья организована следующим образом. В разделе 2 мы рассматриваем функциональные пространства, в которых задано линейное непрерывное представление группы Вейля-Гейзенберга, и определяем скрученную свертку элементов пространства с элементами сопряженного ему пространства. Здесь же излагаются некоммутативные аналоги некоторых свойств обычной свертки. В разделе 3 вводится основное понятие скрученного свертывателя. Раздел 4 содержит основные теоремы; в нем доказано, что при естественных требованиях к рассматриваемым пространствам справедлива импликации $(2) \Rightarrow(3)$, и получена вытекающая из этого утверждения характеризация алгебры скрученных свертывателей. Раздел 5 посвящен наиболее интересному случаю пространств, инвариантных относительно преобразования Фурье. В разделе 6 общая конструкция иллюстрируется конкретными примерами. Приложение содержит доказательство простого полезного критерия непрерывности действия топологических групп в функциональных пространствах рассматриваемого класса.

\section{2. ГРУППА ВЕЙЛЯ-ГЕЙЗЕНБЕРГА И СКРУЧЕННАЯ СВЕРТКА}

Пусть $E$ - локально выпуклое пространство комплекснозначных функций на $\mathbb{R}^{d}$, a $\theta$ - билинейная симплектическая форма, т. е. невырожденное кососимметрическое скалярное произведение на $\mathbb{R}^{d}$. Предположим, что для всех $s \in \mathbb{R}^{d}$ операторы подкрученного сдвига

$$
\tau_{\theta, s}: g(t) \rightarrow e^{i \theta(s, t) / 2} g(t-s), \quad g \in E,
$$

определены и непрерывны в $E$. Операторы $\tau_{\theta, s}$ реализуют проективное представление группы трансляций, которому соответствует линейное представление ее центрального расширения, порождаемого исходной симплектической структурой, т. е. группы Вейля-Гейзенберга, состоящей из элементов вида $a=(\alpha, s)$, где $\alpha \in \mathbb{R}$ и $s \in \mathbb{R}^{d}$, со следующим законом умножения:

$$
a_{1} a_{2}=\left(\alpha_{1}+\alpha_{2}+\frac{1}{2} \theta\left(s_{1}, s_{2}\right), s_{1}+s_{2}\right) .
$$

Предположим еще, что пространство $E$ инвариантно относительно комплексного сопряжения $g \rightarrow g^{*}$. Тогда наряду с представлением $a \rightarrow e^{i \alpha} \tau_{\theta, s}$ в $E$ реализуется и сопряженное представление $a \rightarrow e^{-i \alpha} \bar{\tau}_{\theta, s}$, где

$$
\bar{\tau}_{\theta, s}: g(t) \rightarrow e^{-i \theta(s, t) / 2} g(t-s) .
$$

Если, кроме того, $E$ инвариантно относительно отражения координат $g(t) \rightarrow \check{g}(t)=$ $g(-t)$, то для любых $g \in E$ и $v \in E^{\prime}$ корректно определены функции

$$
\left(v \circledast_{\theta} g\right)(s):=\left\langle v, e^{i \theta(s, \cdot) / 2} g(s-\cdot)\right\rangle, \quad\left(g \circledast_{\theta} v\right)(s):=\left\langle v, e^{-i \theta(s, \cdot) / 2} g(s-\cdot)\right\rangle,
$$


которые мы называем скрученными свертками функции $g$ и функционала $v$.

ЗАмЕчАниЕ 1. В определении группы Вейля-Гейзенберга симплектическая форма обычно предполагается стандартной, задаваемой матрицей $J=\left(\begin{array}{cc}0 & I_{n} \\ -I_{n} & 0\end{array}\right)$, где $2 n=d$, но для любой другой формы $\theta$ группа, очевидно, будет той же самой с точностью до изоморфизма, поскольку в каждом симплектическом пространстве существует симплектический базис [27]. Скрученная свертка в формулировке ВигнераВейля квантовой механики на линейном фазовом пространстве задается матрицей $\hbar J$, где постоянная Планка $\hbar$ играет роль параметра некоммутативной деформации.

Хотя операторы (4), (5) и функции (6) несут индекс $\theta$, мы не будем выписывать его в том случае, если это не может вызвать недоразумений. В дальнейшем мы считаем, что $E$ является плотным векторным подпространством пространства Шварца $S$, но определение (6) содержательно и в более общей ситуации, например когда $E$ непрерывно и плотно вложено в $L^{2}$, - это обеспечивает наличие канонического вложения $E \rightarrow E^{\prime}$, и тогда формулы (6) расширяют операцию скрученной свертки элементов самого пространства $E$. Как обычно, мы обозначаем через $C\left(\mathbb{R}^{d}\right)$ пространство непрерывных функций на $\mathbb{R}^{d}$, снабженное топологией равномерной сходимости на компактных множествах.

ПрЕДЛОЖЕНИЕ 1. Пусть Е - локально выпуклое пространство комплекснозначных функций на $\mathbb{R}^{d}$. Если отражение $g \rightarrow \check{g}$ и операторы (4), (5) действуют в Е непрерывно, то функиии (6) принадлежат $C\left(\mathbb{R}^{d}\right)$. Если, кроме того, Е бочечно, то отображения $(g, v) \rightarrow v \circledast g u(g, v) \rightarrow g \circledast v$ из $E \times E^{\prime}$ в $C\left(\mathbb{R}^{d}\right)$ раздельно непрерывны.

ДокАЗАТЕЛьство. Формулы (6) могут быть записаны как

$$
(v \circledast g)(s)=\left\langle v, \tau_{s} \check{g}\right\rangle, \quad(g \circledast v)(s)=\left\langle v, \bar{\tau}_{s} \check{g}\right\rangle .
$$

Непрерывность действия $\tau_{s}$ в $E$ означает, что отображение $\mathbb{R}^{d} \times E \rightarrow E:(s, g) \rightarrow \tau_{s} g$ непрерывно. Поэтому непрерывность функций (6) по $s$ при сделанных предположениях очевидна. Если $g$ фиксировано и $s$ пробегает компакт $K$ в $\mathbb{R}^{d}$, то $\tau_{s} \check{g}$ пробегает компакт $Q$ в $E$. Значит, отображение $v \rightarrow v \circledast g$ непрерывно относительно сильной топологии $E^{\prime}$ по ее определению как топологии равномерной сходимости на ограниченных множествах. Аналогичным образом, непрерывно отображение $v \rightarrow g \circledast v$. Пусть теперь $v$ фиксировано. Множество операторов $\tau_{s}, s \in K$, поточечно ограничено и, если $E$ бочечно, оно равностепенно непрерывно по обобщенной теореме Банаха-Штейнхауза ([26], § III.4.2). Следовательно, для любой окрестности нуля $U$ в $E$ найдется окрестность $V$ такая, что $\tau_{s} \check{g} \in U$ при всех $g \in V$ и всех $s \in K$. Взяв в качестве $U$ окрестность, на которой функционал $v$ ограничен числом $\epsilon>0$, мы заключаем, что отображение $g \rightarrow v \circledast g$ непрерывно. Аналогичным образом проверяется, что непрерывно отображение $g \rightarrow g \circledast v$. Предложение доказано.

ЗАмЕчАниЕ 2. Наши основные теоремы будут касаться полных ядерных пространств. Такие пространства бочечны в том и только том случае, если они рефлексивны, и тогда они являются монтелевскими [26]. Для монтелевских функциональных пространств можно указать легко проверяемые достаточные условия 
непрерывности действия топологической группы преобразований, которые сформулированы ниже в приложении в виде леммы 1.

Операторы $\tau_{s}$ являются автоморфизмами пространства $C\left(\mathbb{R}^{d}\right)$, и легко видеть, что линейное отображение $L_{v}: g \rightarrow v \circledast g$ коммутирует с ними. Аналогичным образом, отображение $R_{v}: g \rightarrow g \circledast v$ коммутирует со всеми операторами $\bar{\tau}_{s}$.

ПреДЛОЖЕНИЕ 2. Пусть Е удовлетворяет условиям предложения 1 и пусть $A$ - линейное непрерывное отображение из $E$ в $C\left(\mathbb{R}^{d}\right)$. Если $A$ коммутирует со всеми операторами $\tau_{s}$ (со всеми $\left.\bar{\tau}_{s}\right)$, то существует единственный функционал $v \in E^{\prime}$ такой, что $A g=v \circledast g(A g=g \circledast v)$ для всех $g \in E$.

ДоказАтельство. Аргументация аналогична используемой в теореме 4.2.1 в [28], относящейся к обычной свертке и к пространству $E=C_{0}^{\infty}\left(\mathbb{R}^{d}\right)$. Изложим ее для случая перестановочности $A$ со всеми $\tau_{s}$. Если искомый функционал $v$ существует, то $\langle v, \check{g}\rangle=(A g)(0)$ для всех $g \in E$, поэтому он единствен. Обозначим через $v$ линейную форму $g \rightarrow(A \check{g})(0)$. Ясно, что $v \in E^{\prime}$ благодаря непрерывности оператора $A$ и отражения $g \rightarrow \check{g}$. Кроме того, $(v \circledast g)(0)=\langle v, \check{g}\rangle=(A g)(0)$. Используя перестановочность $A$ с $\tau_{s}$, получаем

$$
(A g)(-s)=\left(\tau_{s} A g\right)(0)=\left(A \tau_{s} g\right)(0)=\left(v \circledast \tau_{s} g\right)(0)=(v \circledast g)(-s) \quad \text { для всех } s \in \mathbb{R}^{d} .
$$

Значит, $A g=v \circledast g$ для всех $g \in E$. Во втором случае рассуждение аналогично. Предложение доказано.

\section{3. СКРУЧЕННЫЕ СВЕРТЫВАТЕЛИ}

Далее мы предполагаем, что $E$ состоит из непрерывных функций, причем вложение $E \rightarrow C\left(\mathbb{R}^{d}\right)$ непрерывно, и что выполнены условия предложения 1 . Определим пространства $\mathcal{C}_{\theta, \mathrm{L}}(E)$ и $\mathcal{C}_{\theta, \mathrm{R}}(E)$ левых и правых $\circledast$-свертывателей для $E$, составив их из тех функционалов $v \in E^{\prime}$, для которых $v \circledast_{\theta} g \in E$ и, соответственно, $g \circledast_{\theta} v \in E$ при всех $g \in E$, причем отображения $g \rightarrow v \circledast_{\theta} g$ и $g \rightarrow g \circledast_{\theta} v$ непрерывны.

Если к $E$ применима теорема о замкнутом графике, то требование непрерывности соблюдается здесь автоматически и его можно исключить из данного определения, поскольку эти отображения имеют замкнутые графики. Действительно, если $g_{\gamma} \rightarrow g$ и $v \circledast g_{\gamma} \rightarrow h$ в $E$, то $v \circledast g_{\gamma} \rightarrow h$ в $C\left(\mathbb{R}^{d}\right)$ и $v \circledast g=h$ согласно предложению 1. Из предложения 2 следует, что имеется взаимно однозначное соответствие между элементами $\mathcal{C}_{\mathrm{L}}(E)$ и непрерывными линейными отображениями $E \rightarrow E$, коммутирующими со всеми $\tau_{s}$. Обозначая через $\mathcal{L}(E)$ алгебру всех непрерывных операторов из $E$ в $E$, снабженную топологией равномерной сходимости на ограниченных множествах, мы заключаем, что $\mathcal{C}_{\mathrm{L}}(E)$ можно отождествить с ее замкнутой подалгеброй. Если $v_{1}, v_{2} \in \mathcal{C}_{\mathrm{L}}(E)$, то через $v_{1} \circledast v_{2}$ мы обозначаем тот элемент в $\mathcal{C}_{\mathrm{L}}(E)$, который согласно предложению 2 соответствует суперпозиции операторов $g \rightarrow v_{1} \circledast g$ и $g \rightarrow v_{2} \circledast g$. В явном виде имеем

$$
\left\langle v_{1} \circledast v_{2}, \check{g}\right\rangle=\left(v_{1} \circledast\left(v_{2} \circledast g\right)\right)(0), \quad g \in E .
$$

Естественной топологией $\mathcal{C}_{\mathrm{L}}(E)$ является та, что индуцирована из $\mathcal{L}(E)$. Аналогичным образом, $\mathcal{C}_{\mathrm{R}}(E)$ можно отождествить с подалгеброй операторов, коммутирующих со всеми $\bar{\tau}_{s}$. Для элементов $\mathcal{C}_{\mathrm{R}}(E)$ скрученная свертка определяется формулой

$$
\left\langle v_{1} \circledast v_{2}, \check{g}\right\rangle=\left(\left(g \circledast v_{1}\right) \circledast v_{2}\right)(0), \quad g \in E .
$$


Если $E$ имеет структуру топологической алгебры относительно произведения (1), то алгебры $\mathcal{C}_{\mathrm{L}}(E)$ и $\mathcal{C}_{\mathrm{R}}(E)$ являются ее расширениями. Обе они унитальны, поскольку $\delta$-функция Дирака служит для них единицей. Каждый $\circledast$-свертыватель в $E$ порождает по дуальности соответствующие операции в сопряженном пространстве $E^{\prime}$, определяемые формулами

$$
\begin{aligned}
\left\langle w \circledast v_{1}, g\right\rangle & =\left\langle w, \check{v}_{1} \circledast g\right\rangle, \quad\left\langle v_{2} \circledast w, g\right\rangle=\left\langle w, g \circledast \check{v}_{2}\right\rangle, \\
w & \in E^{\prime}, \quad v_{1} \in \mathcal{C}_{\mathrm{L}}(E), \quad v_{2} \in \mathcal{C}_{\mathrm{R}}(E),
\end{aligned}
$$

что является дальнейшим расширением исходной операции в пространстве $E$.

Если пространство $E$ инвариантно относительно комплексного сопряжения, то инволюция $g \rightarrow g^{*}$ в $E$ индуцирует инволюцию в $E^{\prime}$; при этом $(v \circledast g)^{*}=g^{*} \circledast v^{*}$, и тем самым имеется канонический антилинейный изоморфизм между $\mathcal{C}_{\mathrm{L}}(E)$ и $\mathcal{C}_{\mathrm{R}}(E)$. Рассмотрим теперь пересечение

$$
\mathcal{C}_{\theta}(E)=\mathcal{C}_{\theta, \mathrm{L}}(E) \cap \mathcal{C}_{\theta, \mathrm{R}}(E)
$$

Естественной топологией $\mathcal{C}_{\theta}(E)$ является верхняя грань топологий, индуцированных из $\mathcal{C}_{\theta, \mathrm{L}}(E)$ и $\mathcal{C}_{\theta, \mathrm{R}}(E)$. Если $E \cap \mathcal{C}_{\theta}(E)$ плотно в $\mathcal{C}_{\theta}(E)$ по этой топологии и $E$ состоит из ограниченных, непрерывных, интегрируемых функций, то $\mathcal{C}_{\theta}(E)$ имеет каноническую структуру инволютивной унитальной алгебры относительно $\circledast$-произведения, поскольку тогда формулы (7) и (8) с $v_{1}, v_{2} \in \mathcal{C}_{\theta}(E)$ определяют один и тот же функционал. Действительно, используя теорему Фубини, легко проверить, что при указанных условиях и для $v_{1}, v_{2} \in E$ интегралы, представляющие правые части этих формул, совпадают и наше утверждение доказывается предельным переходом.

\section{4. ОСНОВНЫЕ ТЕОРЕМЫ}

Несколько слов об используемой ниже терминологии. Пусть $E$ - полное ядерное локально выпуклое пространство. Если оно является линейным подпространством другого локально выпуклого пространства $E_{0}$ и тождественное отображение $E \rightarrow E_{0}$ непрерывно, то мы говорим, что $E$ - полное ядерное подпространство в $E_{0}$. Нам понадобится следующее вспомогательное утверждение.

ПрЕДЛОЖЕНИЕ 3. Пусть Е - полное ядерное подпространство пространства Швариа $S\left(\mathbb{R}^{d}\right)$. Тогда $Е \widetilde{\otimes}$ Е является полным ядерным подпространством в $S\left(\mathbb{R}^{2 d}\right)$.

ДокАзАтельство. Напомним, что пополненное проективное тензорное произведение ядерных пространств ядерно [26]. Поскольку $S\left(\mathbb{R}^{2 d}\right)=S\left(\mathbb{R}^{d}\right) \widetilde{\otimes} S\left(\mathbb{R}^{d}\right)$, имеется естественное непрерывное линейное отображение $\iota: E \otimes E \rightarrow S\left(\mathbb{R}^{2 d}\right)$, и надо убедиться лишь в том, что его продолжение $\tilde{\iota}$ по непрерывности на $E \widetilde{\otimes} E$ инъективно. Согласно теореме Гротендика ([26], § IV.9.4), если $E$ полно и ядерно, $E \widetilde{\otimes} E$ можно отождествить с пространством $\mathcal{B}_{e}\left(E_{\sigma}^{\prime}, E_{\sigma}^{\prime}\right)$ раздельно непрерывных билинейных форм на $E_{\sigma}^{\prime} \times E_{\sigma}^{\prime}$, где индексы $\sigma$ и $е$ соответственно означают, что $E^{\prime}$ наделено слабой топологией, а $\mathcal{B}$ - топологией биравностепенной сходимости. Каноническое отображение $E \times E \rightarrow B_{e}\left(E_{\sigma}^{\prime}, E_{\sigma}^{\prime}\right)$ сопоставляет паре функций $(f, g)$ билинейную форму

$$
(f \otimes g)(u, v)=\langle u, f\rangle\langle v, g\rangle, \quad u, v \in E^{\prime} .
$$


Обозначим через $j^{\prime}$ отображение, сопряженное вложению $j: E \rightarrow S\left(\mathbb{R}^{d}\right)$. Используя указанное отождествление также и для пространства Шварца, мы видим, что $\tilde{\iota}$ переводит билинейную форму $\mathbf{b} \in \mathcal{B}_{e}\left(E_{\sigma}^{\prime}, E_{\sigma}^{\prime}\right)$ в билинейную форму на $S^{\prime} \times S^{\prime}$, значение которой на паре распределений $u, v \in S^{\prime}$ есть $\mathbf{b}\left(j^{\prime}(u), j^{\prime}(v)\right)$. Поскольку $j-$ инъекция, $j^{\prime}\left(S^{\prime}\right)$ плотно в $E_{\sigma}^{\prime}$ и, значит, $\tilde{\iota}(\mathbf{b})=0$ влечет $\mathbf{b}=0$, что завершает доказательство.

Если выполнены условия предложения 3 , мы будем наряду с $E \widetilde{\otimes} E$ использовать для этого пространства обозначение $E\left(\mathbb{R}^{2 d}\right)$. Простое доказательство теоремы о ядре, обобщающей знаменитую теорему Шварца для $S\left(\mathbb{R}^{d}\right)$ на любое полное ядерное бочечное пространство, дано в работе [29]. Оно развивает конструкцию, предложенную Гротендиком ([30], гл. 2, теорема 13). На практике $E\left(\mathbb{R}^{2 d}\right)$ определяется теми же ограничениями, что и $E\left(\mathbb{R}^{d}\right)$, но только налагаемыми на функции от удвоенного числа переменных (см. примеры в разделе 6).

Хорошо известно, что свертка любого распределения умеренного роста с пробной функцией из пространства Шварца $S$ является мультипликатором для этого пространства относительно поточечного умножения. Следующая теорема устанавливает аналог этого свойства для более широких классов обобщенных функций и показывает, что оно сохраняется при некоммутативной деформации свертки. Для любого локально выпуклого подпространства $E \subset S$ непрерывная функция $\mu$ называется его мультипликатором относительно поточечного умножения, если $\mu g \in E$ при всех $g \in E$ и отображение $g \rightarrow \mu g$ непрерывно ${ }^{3)}$. Множество всех таких мультипликаторов мы обозначаем через $M(E)$ и снабжаем топологией, индуцированной из операторной алгебры $\mathcal{L}(E)$.

Теорема 1. Пусть $E$ - полное ядерное бочечное подпространство в $S\left(\mathbb{R}^{d}\right)$, a $\theta(s, t)$ - симплектическая форма на $\mathbb{R}^{d}$. Предположим, что $e^{-i \theta / 2} \in M(E \widetilde{\otimes} E)$ $u$ инволютивное преобразование $h(s, t) \rightarrow h(s, s-t)$ есть непрерывный автоморбизм пространства $E\left(\mathbb{R}^{2 d}\right)=E \widetilde{\otimes} E$. Тогда $E$ является алгеброй относительно $\circledast_{\theta}$-произведения и это произведение раздельно непрерывно; функиии $v \circledast_{\theta} g u g \circledast_{\theta} v$ корректно определены и принадлежат $M(E)$ nри любых $g \in E u v \in E^{\prime}$. Отображения $(g, v) \rightarrow v \circledast_{\theta} g u(g, v) \rightarrow g \circledast_{\theta} v$ uз $E \times E^{\prime}$ в $M(E)$ также раздельно непрерьвны.

ДокАзАтЕльство. Покажем сначала, что если $h \in E\left(\mathbb{R}^{2 d}\right)$, то при каждом фиксированном $s$ функция $h(s, t)$ по переменной $t$ принадлежит $E$. Как и при доказательстве предложения 3 , отождествляем $h$ с билинейным раздельно непрерывным функционалом $\mathbf{h}$ на $E_{\sigma}^{\prime} \times E_{\sigma}^{\prime}$. Заметим, что

$$
h(s, t)=\mathbf{h}\left(\delta_{s}, \delta_{t}\right)
$$

где функционал $\delta_{s} \in E^{\prime}$ определяется формулой $\left\langle\delta_{s}, f\right\rangle=f(s)$. Действительно, из (11) следует (12) для линейных комбинаций функций вида $h=f \otimes g$, а по непрерывности (12) соблюдается и для любого элемента в $E\left(\mathbb{R}^{2 d}\right)$. Линейное отображение $E^{\prime} \rightarrow \mathbb{C}: v \rightarrow \mathbf{h}\left(\delta_{s}, v\right)$ непрерывно по слабой топологии $\sigma\left(E^{\prime}, E\right)$. Значит, существует

3) Условие непрерывности соблюдается автоматически, если к $E$ применима теорема о замкнутом графике. 
функция $h_{s} \in E=\left(E_{\sigma}^{\prime}\right)^{\prime}$ такая, что $\left\langle v, h_{s}\right\rangle=\mathbf{h}\left(\delta_{s}, v\right)$ при всех $v \in E^{\prime}$. Подставляя сюда $v=\delta_{t}$, мы получаем $h_{s}(t)=h(s, t)$. Ясно, что отображение $h \rightarrow h_{s}$ непрерывно при наделении $E\left(\mathbb{R}^{2 d}\right)$ и $E$ слабыми топологиями. Далее, легко видеть, что функция $s \rightarrow\langle v, h(s, \cdot)\rangle=\mathbf{h}\left(\delta_{s}, v\right)$ принадлежит $E$. Действительно, линейное отображение $u \rightarrow \mathbf{h}(u, v)$ непрерывно по топологии $\sigma\left(E^{\prime}, E\right)$, и, значит, существует функция $h_{v} \in E$ такая, что $\left\langle u, h_{v}\right\rangle=\mathbf{h}(u, v)$ при всех $u \in E^{\prime}$. При $u=\delta_{s}$ это равенство принимает вид $h_{v}(s)=\langle v, h(s, \cdot)\rangle$. Отображение $E^{\prime} \rightarrow E: v \rightarrow h_{v}$ также слабо непрерывно. Кроме того, слабо непрерывна зависимость $h_{v}$ от $h$.

Применим изложенное рассмотрение к функции $h(s, t)=f(s) g(s-t)$, где $f, g \in E$. По условию теоремы она принадлежит $E\left(\mathbb{R}^{2 d}\right)$. Фиксируем точку $s \in \mathbb{R}^{d}$ и выберем $f$ так, чтобы $f(s)=1$. В силу сказанного выше функция $t \rightarrow g(s-t)$ принадлежит $E$ и отображение $E \rightarrow E: g(t) \rightarrow g(s-t)$ слабо непрерывно. Поскольку любое бочечное пространство есть пространство Макки, отсюда вытекает, что это отображение непрерывно ([26], § IV.7.4). Так как $s$ можно взять любым, мы видим, что отражения и сдвиги являются непрерывными операторами в пространстве $E$. Аналогичные соображения в применении к $f(s) e^{-i \theta(s, t) / 2} g(s-t)$ показывают, что функция $t \rightarrow e^{-i \theta(s, t) / 2} g(s-t)$ принадлежит $E$, причем отображение $g(t) \rightarrow e^{-i \theta(s, t) / 2} g(s-t)$ непрерывно. Следовательно, подкрученные сдвиги (4) и (5) также являются непрерывными операторами в $E$ и применимы определения функций $v \circledast g$ и $g \circledast v$, данные в разделе 2. Далее, поскольку $f(s)\left\langle v, e^{ \pm i \theta(s, \cdot) / 2} g(s-\cdot)\right\rangle \in E$ для любой функции $f \in E$, функции $\left\langle v, \tau_{s} \check{g}\right\rangle$ и $\left\langle v, \bar{\tau}_{s} \check{g}\right\rangle$ непрерывны по $s$ при всяком $v \in E^{\prime}$. Значит, множества $\left\{\tau_{s} \check{g}:|s| \leqslant 1\right\}$ и $\left\{\bar{\tau}_{s} \check{g}:|s| \leqslant 1\right\}$, где $g$ фиксировано, слабо ограничены. Тогда они ограничены и в исходной топологии $E([26], \S \mathrm{IV} .3 .2)$, и согласно лемме 1 , доказанной в приложении, соответствующие представления группы Вейля-Гейзенберга в пространстве $E$ непрерывны. При фиксированных $v$ и $g$ функции $f \cdot(v \circledast g)$ и $f \cdot(g \circledast v)$ слабо непрерывны по $f$, и, значит, умножение на $v \circledast g$ и умножение на $g \circledast v$ являются непрерывными операторами из $E$ в $E$. Мы заключаем, что скрученные свертки принадлежат $M(E)$. Кроме того, снова в силу сказанного выше, при любом $u \in E^{\prime}$ функция $t \rightarrow\left\langle u, f(\cdot) e^{-i \theta(\cdot, t) / 2} g(t-\cdot)\right\rangle$ принадлежит $E$. В частности,

$$
\int f(s) e^{-i \theta(s, t) / 2} g(t-s) d s=f \circledast g \in E .
$$

Таким образом, $E$ является алгеброй относительно скрученной свертки. Отображения $f \rightarrow f \circledast g$ и $g \rightarrow f \circledast g$, очевидно, слабо непрерывны, а поэтому непрерывны и относительно исходной топологии $E$.

Осталось убедиться, что отображения $(g, v) \rightarrow v \circledast g$ и $(g, v) \rightarrow g \circledast v$ из $E \times E^{\prime}$ в $M(E)$ раздельно непрерывны. Пусть $v$ фиксировано. Покажем, что для любой окрестности нуля $U$ и любого ограниченного множества $Q$ в $E$ найдется окрестность нуля $W$ такая, что $f \cdot(v \circledast g) \in U$ при всех $f \in Q$ и всех $g \in W$. По определению топологии биравностепенной сходимости базис окрестностей нуля в $E \widetilde{\otimes} E=\mathcal{B}_{e}\left(E_{\sigma}^{\prime}, E_{\sigma}^{\prime}\right)$ образует множества вида

$$
\mathcal{U}_{U, V}=\left\{\mathbf{b} \in \mathcal{B}\left(E_{\sigma}^{\prime}, E_{\sigma}^{\prime}\right): \sup _{u \in U^{\circ}, v \in V^{\circ}}|\mathbf{b}(u, v)| \leqslant 1\right\}
$$

где $U$ и $V$ пробегают базис замкнутых абсолютно выпуклых окрестностей в $E$, а $U^{\circ}, V^{\circ}$ обозначают их поляры, т. е. $U^{\circ}=\left\{u \in E^{\prime}: \sup _{f \in U}|\langle u, f\rangle| \leqslant 1\right\}$. Функционал $v$ ограничен единицей на некоторой окрестности нуля, которую мы возьмем 
в качестве $V$ в (13). По условиям теоремы для любого множества $\mathcal{U}_{U, V}$ найдутся окрестности $U_{1}, V_{1}$ такие, что

$$
h(s, t)=f(s) e^{i \theta(s, t) / 2} g(s-t) \in \mathcal{U}_{U, V}
$$

при всех $f \in U_{1}$ и $g \in V_{1}$. Для ограниченного множества $Q$ найдется $\delta>0$ такое, что $Q \subset \delta U_{1}$. Положим $W=\delta V_{1}$. Тогда при всех $u \in U^{\circ}, f \in Q$ и $g \in W$ мы имеем

$$
|\mathbf{h}(u, v)|=|\langle u, f(v \circledast g)\rangle| \leqslant 1 .
$$

Иными словами, $f \cdot(v \circledast g) \in U^{\circ \circ}=U$, что доказывает непрерывность отображения $E \rightarrow M(E): g \rightarrow v \circledast g$.

Пусть теперь $g$ фиксировано и последовательность $v_{n}$ стремится к нулю в $E^{\prime}$. Тогда последовательность мультипликаторов $v_{n} \circledast g \rightarrow 0$ в топологии простой сходимости $L(E)$, а поскольку $E$ - монтелевское пространство (как уже было сказано в замечании 2), то и в топологии ограниченной сходимости (см. [26], § III.4.6). Таким образом, отображение $E^{\prime} \rightarrow \mathcal{L}(E): v \rightarrow v \circledast g$ секвенциально непрерывно. Остается показать, что пространство $E^{\prime}$ борнологично, поскольку для таких пространств секвенциальная непрерывность линейного отображения в любое локально выпуклое пространство влечет его непрерывность ([26], § II.8.3). С этой целью напомним, что всякое полное ядерное пространство $E$ представимо как проективный предел некоторого семейства гильбертовых пространств $E_{\gamma}$. Этот предел можно считать приведенным, и сопряженное пространство $E^{\prime}$ в топологии Макки $\tau\left(E^{\prime}, E\right)$ есть индуктивный предел семейства пространств $E_{\gamma}^{\prime}$, снабженных топологиями Макки $\tau\left(E_{\gamma}^{\prime}, E_{\gamma}\right)$ ([26], § IV.4.4). Поскольку монтелевские и гильбертовы пространства рефлексивны, сильная топология на сопряженных им пространствах совпадает с топологией Макки. Значит, $E^{\prime}$ борнологично как индуктивный предел нормированных пространств. Мы заключаем, что отображение $E^{\prime} \rightarrow M(E): v \rightarrow v \circledast g$ непрерывно. Аналогичная аргументация для $g \circledast v$ завершает доказательство.

СлЕДСТвИЕ 1. Если выполнены предположения теоремы 1, то определение (6) равносильно определению произведений $v \circledast g u g \circledast v$ по дуальности и для всех $v \in E^{\prime}$ и всех $g, f \in E$ справедливы равенства

$$
(v \circledast g) \circledast f=v \circledast(g \circledast f), \quad(g \circledast v) \circledast f=g \circledast(v \circledast f), \quad g \circledast(f \circledast v)=(g \circledast f) \circledast v .
$$

ДокАзАтЕльство. Для всякой топологической алгебры $(E, \circledast)$ скрученная свертка функции $g \in E$ с функционалом $v \in E^{\prime}$ определяется по дуальности формулами

$$
\langle v \circledast g, f\rangle=\langle v, \check{g} \circledast f\rangle, \quad\langle g \circledast v, f\rangle=\langle v, f \circledast \check{g}\rangle, \quad f \in E .
$$

Тогда отображения $v \rightarrow v \circledast g$ и $v \rightarrow g \circledast v$ из $E^{\prime}$ в $E^{\prime}$ непрерывны, поскольку являются сопряженными к непрерывным отображениям $f \rightarrow \check{g} \circledast f$ и $f \rightarrow f \circledast \check{g}$. Легко видеть, что при $v \in E$ правые части в равенствах (15) совпадают с результатом интегрирования функций (6) с пробной функцией $f$. Надо показать, что это верно и при $v \in E^{\prime}$. Для этого заметим, что при условиях теоремы 1 пространство $E$ плотно в $E^{\prime}$. Действительно, как показано при доказательстве теоремы $1, E$ содержит вместе с функцией $g(t)$ и все ее сдвиги $g(t-s)$, а также все произведения $e^{i \theta(s, t) / 2} g(t)$, $s \in \mathbb{R}^{d}$. Согласно [23] (§ IV.8.4) отсюда следует, что $E$ имеет достаточно большой 
запас функций в том смысле, что для любой локально интегрируемой функции $\varphi$ из сходимости интеграла $\int \varphi(t) f(t) d t$ и равенства его нулю для всех $f \in E$ вытекает, что $\varphi(t) \equiv 0$. Значит, каноническое отображение $E \rightarrow E^{\prime}$ инъективно, и, поскольку $E$ рефлексивно, по теореме Хана-Банаха это отображение имеет плотный образ, что и утверждалось. Далее, всякий мультипликатор $m \in M(E)$ определяет функционал $\mu \in E^{\prime}$ по формуле $\langle\mu, f\rangle=\int m(t) f(t) d t$, причем отображение

$$
M(E) \rightarrow E^{\prime}: m \rightarrow \mu
$$

инъективно в силу тех же соображений и непрерывно по определению топологии этих пространств. Аппроксимируя функционал $v \in E^{\prime}$ функциями $v_{\gamma} \in E$, используя непрерывность отображений $v \rightarrow v \circledast g, v \rightarrow g \circledast v$ из $E^{\prime}$ в $M(E)$ и переходя к пределу в равенстве

$$
\int\left(v_{\gamma} \circledast g\right)(t) f(t) d t=\left\langle v_{\gamma}, \check{g} \circledast f\right\rangle
$$

и в аналогичном равенстве для $g \circledast v_{\gamma}$, мы заключаем, что отображение (16) устанавливает взаимно однозначное соответствие между функциями, определяемыми формулами (6), и функционалами, определяемыми посредством (15). Формулы (14) следуют из ассоциативности алгебры $(E, \circledast)$ также по непрерывности, что завершает доказательство.

Теперь заметим, что при условиях теоремы 1 пространство $E$ является алгеброй относительно поточечного умножения. Действительно, при любых $f, g \in E$ функция $\left.f(s) g(s-t)\right|_{t=0}$ принадлежит $E$, причем из доказательства теоремы 1 следует, что отображение $(f, g) \rightarrow f \cdot g$ раздельно непрерывно. Таким образом, имеется каноническое вложение $E \rightarrow M(E)$. В дальнейшем мы считаем, что $E$ плотно в $M(E)$. Это условие обычно предполагается при рассмотрении мультипликаторов топологических алгебр, а в работе [24] оно включено в определение $M(E)$. Если оно выполнено, то алгебра $M(E)$ канонически изоморфна замыканию в $\mathcal{L}(E)$ множества операторов умножения на элементы $E$.

Теорема 2. Если выполнены предположения теоремы 1 и E плотно в $M(E)$, то сопряженное $\kappa M(E)$ пространство $M^{\prime}(E)$ содержится в $\mathcal{C}_{\theta}(E)$.

ДоказАтельство. Пусть $Q$ - ограниченное множество в пространстве $E$. Множество непрерывных отображений $g \rightarrow f \cdot g, f \in Q$, поточечно ограничено. По теореме Банаха-Штейнхауза отсюда следует, что для всякой окрестности нуля $U$ в $E$ найдется окрестность $V$ такая, что $f \cdot g \in U$ при всех $g \in V, f \in Q$. Это означает, что естественное вложение $E \rightarrow M(E)$ непрерывно. Следовательно, определено и непрерывно сопряженное отображение $M^{\prime}(E) \rightarrow E^{\prime}$. Из предположения о плотности $E$ в $M(E)$ вытекает, что это отображение инъективно. Поэтому если $w \in M^{\prime}(E)$, то $w \in E^{\prime}$ и $w \circledast g \in M(E)$ согласно теореме 1 . Покажем, что $w \circledast g \in E$. По следствию 1 мы имеем

$$
(w \circledast g) \circledast f=w \circledast(g \circledast f) \quad \text { при всех } g, f \in E .
$$

Равенство этих сверток в нуле может быть записано как

$$
\langle w \circledast g, \check{f}\rangle=\langle\check{w}, g \circledast f\rangle .
$$


Пусть $L_{g}^{\prime}$ - линейное отображение из $M^{\prime}(E)$ в $E$, сопряженное непрерывному отображению $L_{g}: v \rightarrow g \circledast v$. Тогда

$$
\left\langle v, L_{g}^{\prime} \check{w}\right\rangle=\langle\check{w}, g \circledast v\rangle \quad \text { при всех } v \in E^{\prime} .
$$

При $v=f$ правые части (17) и (18) совпадают, а левая часть (18) записывается как $\int\left(L_{g}^{\prime} \check{w}\right)(t) f(t) d t$. Поскольку $f$ - произвольный элемент пространства $E$, которое достаточно богато функциями, мы заключаем, что функция $(w \circledast g)(t)$ совпадает с функцией $\left(L_{g}^{\prime} \check{w}\right)(-t)$, принадлежащей пространству $E$. Осталось показать, что отображение $E \rightarrow E: g \rightarrow w \circledast g$ непрерывно. По теореме 1 при любом фиксированном $v \in E^{\prime}$ отображение $E \rightarrow M(E): g \rightarrow g \circledast v$ непрерывно. Тем более оно непрерывно при наделении $E$ и $M(E)$ слабыми топологиями. Поэтому числовая функция $g \rightarrow\left\langle\breve{w}, L_{g} v\right\rangle$ непрерывна по топологии $\sigma\left(E, E^{\prime}\right)$. Следовательно, отображение $E \rightarrow E: g \rightarrow L_{g}^{\prime} \check{w}=w \circledast g$ слабо непрерывно. Поскольку $E$ - пространство Макки, это отображение непрерывно и относительно его исходной топологии. Мы заключаем, что $w \in \mathcal{C}_{\mathrm{L}}(E)$. Аналогичным образом, $w \in \mathcal{C}_{\mathrm{R}}(E)$. Теорема доказана.

Из теоремы 2 вытекает соответствующий результат для пространства $F$, которое двойственно $E$ относительно преобразования Фурье, т. е. $E=\widehat{F}$. Преобразование Фурье функции $f$ мы обозначаем через

$$
\hat{f}(s):=\int f(x) e^{-i x \cdot s} d x
$$

Для любых функций $f_{1}, f_{2}$ из пространства Шварца $S$ мы имеем соотношение

$$
\left(\widehat{f_{1} \star_{\theta} f_{2}}\right)=(2 \pi)^{-d} \hat{f}_{1} \circledast{ }_{\theta} \hat{f}_{2},
$$

которое можно взять за определение мойаловского произведения элементов $S$. Поэтому если $E$ удовлетворяет условиям теорем 1 и 2 , то $F$ является алгеброй относительно произведения $\star_{\theta}$ с раздельно непрерывным умножением. Это произведение, очевидно, имеет единственное продолжение по непрерывности на тот случай, когда один из сомножителей принадлежит сопряженному пространству, и если $u \in F^{\prime}$, $f \in F$, то

$$
\left(\widehat{u \star_{\theta} f}\right)=(2 \pi)^{-d} \hat{u} \circledast{ }_{\theta} \hat{f}, \quad\left(\widehat{f \star_{\theta} u}\right)=(2 \pi)^{-d} \hat{f} \circledast_{\theta} \hat{u} .
$$

Кроме того, справедливы формулы

$$
\left\langle u \star_{\theta} g, f\right\rangle=\left\langle u, g \star_{\theta} f\right\rangle, \quad\left\langle g \star_{\theta} u, f\right\rangle=\left\langle u, f \star_{\theta} g\right\rangle, \quad f, g \in F, \quad u \in F^{\prime},
$$

дающие продолжение мойаловского произведения на элементы $F^{\prime}$ по дуальности и соответствующие формулам (15). Поскольку $E$ - алгебра по обычному умножению, $F$ является также алгеброй относительно обычной свертки. Обозначим через $C(F)$ соответствующую алгебру обычных свертывателей, а через $\mathcal{M}_{\theta, \mathrm{L}}(F)$, $\mathcal{M}_{\theta, \mathrm{R}}(F)$ - алгебры левых и правых мойаловских мультипликаторов для $F$. Тогда $\widehat{C}(F)=M(\widehat{F}), \widehat{\mathcal{M}}_{\theta, \mathrm{L}}(F)=\mathcal{C}_{\theta, \mathrm{L}}(\widehat{F}), \widehat{\mathcal{M}}_{\theta, \mathrm{R}}(F)=\mathcal{C}_{\theta, \mathrm{R}}(\widehat{F})$ и мы получаем следующий результат.

СледСтвиЕ 2. Если $F$ - пространство функиий, преобразование Фуръе которого $E=\widehat{F}$ удовлетворяет условиям теорем 1 и 2, то пространство $C^{\prime}(F)$ содержстся в $\mathcal{M}_{\theta}(F)=\mathcal{M}_{\theta, \mathrm{L}}(F) \cap \mathcal{M}_{\theta, \mathrm{R}}(F)$. 


\section{5. СЛУЧАЙ ПРОСТРАНСТВ, ИНВАРИАНТНЫХ ОТНОСИТЕЛЬНО ПРЕОБРАЗОВАНИЯ ФУРЬЕ}

Особый интерес представляют фурье-инвариантные функциональные пространства, имеющие структуру алгебры как относительно скрученной свертки, так и относительно произведения Мойала. Напомним, что автогомеоморфизмом топологического пространства называют его взаимно однозначное и взаимно непрерывное отображение на себя.

Теорема 3. Предположим, что для пространства Е выполнены условия теорем 1 и 2. Если, кроме того, преобразование Фуръе и линейные замены координат в $\mathbb{R}^{d}$ являютол автогомеоморфизмами $E$, то оба пространства $M^{\prime}(E)$ и $C^{\prime}(E)$ содержатся как в $\mathcal{C}_{\theta}(E)$, так и в $\mathcal{M}_{\theta}(E)$.

ДокАЗАТЕльСТво. Можно считать, что симплектическая форма $\theta$ задается кососимметрической матрицей $\vartheta$ по формуле $\theta(s, t)=s \cdot \vartheta t$, где точка обозначает обычное скалярное произведение в $\mathbb{R}^{d}$. Пусть $f_{1}, f_{2} \in E$. Из (1) и (19) следует

$$
\begin{aligned}
\left(f_{1} \star_{\theta} f_{2}\right)(x) & =\frac{1}{(2 \pi)^{2 d}} \iiint \hat{f}_{1}(t) f_{2}(y) e^{(i / 2) s \cdot \vartheta t-i(s-t) \cdot y+i s \cdot x} d t d y d s= \\
& =\frac{1}{(2 \pi)^{d}} \iint \hat{f}_{1}(t) f_{2}(y) e^{i t \cdot y} \delta\left(y-x-\frac{1}{2} \vartheta t\right) d t d y= \\
& =\frac{1}{(2 \pi)^{d}} \int \hat{f}_{1}(t) f_{2}\left(x+\frac{1}{2} \theta t\right) e^{i t \cdot x} d t= \\
& =\frac{1}{\pi^{d} \operatorname{det} \theta} \int \hat{f}_{1}\left(-2 \theta^{-1} \xi\right) f_{2}(x-\xi) e^{-2 i x \cdot \theta^{-1} \xi} d \xi .
\end{aligned}
$$

Таким образом,

$$
f_{1} \star_{\vartheta} f_{2}=\frac{1}{\pi^{d} \operatorname{det} \theta}\left(\mathcal{F}_{\vartheta} f_{1}\right) \circledast_{-4 \vartheta^{-1}} f_{2},
$$

где

$$
\left(\mathcal{F}_{\vartheta} f\right)(\xi):=\int f(x) e^{2 i x \cdot \vartheta^{-1} \xi} d x
$$

Аналогичная выкладка дает

$$
f_{1} \star_{\vartheta} f_{2}=\frac{1}{\pi^{d} \operatorname{det} \theta} f_{1} \circledast_{-4 \vartheta^{-1}}\left(\overline{\mathcal{F}}_{\vartheta} f_{2}\right),
$$

где

$$
\left(\overline{\mathcal{F}}_{\vartheta} f\right)(\xi):=\int f(x) e^{-2 i x \cdot \vartheta^{-1} \xi} d x .
$$

Из условий теоремы вытекает, что каждое из симплектических преобразований Фурье $\mathcal{F}_{\vartheta}$ и $\overline{\mathcal{F}}_{\vartheta}$ есть автогомеоморфизм $E$ и, кроме того, $e^{-2 i x \cdot \vartheta^{-1} \xi} \in M(E \widetilde{\otimes} E)$. Поэтому в силу теоремы 1 скрученные свертки $\left(\mathcal{F}_{\vartheta} f\right) \circledast_{-4 \vartheta^{-1}} v$ и $v \circledast_{-4 \vartheta^{-1}}\left(\overline{\mathcal{F}}_{\vartheta} f\right)$ с параметром деформации $-4 \vartheta^{-1}$ корректно определены для всех $f \in E$ и $v \in E^{\prime}$, причем они непрерывны по $v$. Поскольку скрученная свертка и мойаловское произведение единственным образом продолжаются по непрерывности на тот случай, когда один из сомножителей принадлежит сопряженному пространству, мы заключаем, что

$$
f \star_{\vartheta} v=\frac{1}{\pi^{d} \operatorname{det} \theta}\left(\mathcal{F}_{\theta} f\right) \circledast_{-4 \vartheta^{-1}} v, \quad v \star_{\vartheta} f=\frac{1}{\pi^{d} \operatorname{det} \theta} v \circledast_{-4 \vartheta^{-1}}\left(\overline{\mathcal{F}}_{\theta} f\right) .
$$


Из теоремы 1 и формул (22) следует, что $f \star_{\vartheta} v$ и $v \star_{\vartheta} f$ принадлежат $M(E)$ и отображения $(f, v) \rightarrow f \star_{\vartheta} v,(f, v) \rightarrow v \star_{\vartheta} f$ из $E \times E^{\prime}$ в $M(E)$ непрерывны. Дальнейшая аргументация аналогична использованной при доказательстве теоремы 2 . Пусть $w \in M^{\prime}(E) \subset E^{\prime}$. Тогда $w \star_{\vartheta} f \in M(E)$ по теореме 1 . Покажем, что $w \star_{\vartheta} f \in E$. Согласно (20) мы имеем

$$
\left\langle w \star_{\vartheta} f, g\right\rangle=\left\langle w, f \star_{\vartheta} g\right\rangle \quad \text { при всех } g \in E .
$$

Пусть $L_{f}-$ линейное отображение $v \rightarrow f \star_{\theta} v$ из $E^{\prime}$ в $M(E)$. Тогда

$$
\left\langle v, L_{f}^{\prime} w\right\rangle=\left\langle w, f \star_{\vartheta} v\right\rangle \quad \text { при всех } v \in E^{\prime} .
$$

При $v=g$ правые части равенств (23) и (24) совпадают и

$$
\left\langle g, L_{f}^{\prime} w\right\rangle=\int\left(L_{f}^{\prime} w\right)(\xi) g(\xi) d \xi
$$

Следовательно, $w \star \vartheta f$ совпадает с функцией $L_{f}^{\prime} w$, принадлежащей $E$. При любом фиксированном $v \in E^{\prime}$ отображение $E \rightarrow M(E): f \rightarrow f \star_{\vartheta} v$ непрерывно. Тем более оно непрерывно при наделении $E$ и $M(E)$ слабыми топологиями. Поэтому функция $f \rightarrow\left\langle w, L_{f} v\right\rangle$ непрерывна по топологии $\sigma\left(E, E^{\prime}\right)$. Следовательно, отображение $E \rightarrow$ $E: f \rightarrow L_{f}^{\prime} w=w \star_{\vartheta} f$ слабо непрерывно. Поскольку $E-$ пространство Макки, это отображение непрерывно и относительно его исходной топологии. Мы заключаем, что $w \in \mathcal{M}_{\theta, \mathrm{L}}(E)$. Аналогичным образом, $w \in \mathcal{M}_{\theta, \mathrm{R}}(E)$ и, значит, $M^{\prime}(E) \subset \mathcal{M}_{\theta}(E)$. Из этого результата и изоморфизмов $\widehat{M}(E)=C(\widehat{E}), \widehat{\mathcal{M}}_{\theta}(E)=\mathcal{C}_{\theta}(\widehat{E})$, где в данном случае $\widehat{E}=E$, следует, что $C^{\prime}(E) \subset \mathcal{C}_{\theta}(E)$. Теорема доказана.

ЗАмечАниЕ 3. Из формул (22) вытекает, что при условиях теоремы 3 алгебра $\mathcal{M}_{\vartheta}$ и алгебра $\mathcal{C}_{-4 \vartheta^{-1}}(E)=\widehat{\mathcal{M}}_{-4 \vartheta^{-1}}(E)$ состоят из одних и тех же элементов пространства $E^{\prime}$. В частности, при $\theta=\hbar J$, где $J$ - стандартная симплектическая матрица, алгебры $\mathcal{M}_{\hbar J}$ и $\mathcal{C}_{4 \hbar^{-1} J}(E)$ состоят из одних и тех же элементов, а алгебра $\mathcal{M}_{2 J}(E)$ фурье-инвариантна.

\section{6. ПРИМЕРЫ И ЗАКЛЮЧИТЕЛЬНЫЕ ЗАМЕЧАНИЯ}

Многие используемые в теории обобщенных функций пространства являются ядерными пространствами Фреше или сильными сопряженными к ним. Для этих двух классов пространств используются соответственно аббревиатуры FN и DFN. Они обладают дополнительными удобными свойствами. В частности, к ним применима теорема о замкнутом графике в форме Птака; раздельная непрерывность билинейного отображения произведения таких пространств в любое локально выпуклое пространство равносильна для них совместной непрерывности; кроме того, пополненное проективное тензорное произведение двух FN (двух DFN) пространств является FN (DFN) пространством (см. [26], [31]). Отметим, что каждое пространство FN является пространством FS (Фреше-Шварца), а каждое пространство DFN есть пространство DFS. Основные сведения о пространствах FS и DFS можно найти, например, в [32], [33]. Изложенные выше формулировки и доказательства для таких пространств существенно упрощаются. Пространство Шварца $S$ является 
наиболее известным примером пространства FN. K этому классу принадлежат также пространства $\mathscr{S}^{\beta}$, рассматривавшиеся в контексте некоммутативной теории поля в работах [21], [34]. Пространство $\mathscr{S}^{\beta}\left(\mathbb{R}^{d}\right), \beta>0$, определяется как проективный предел proj $\lim _{N \rightarrow \infty, B \rightarrow 0} S_{N}^{\beta, B}\left(\mathbb{R}^{d}\right)$, где $S_{N}^{\beta, B}\left(\mathbb{R}^{d}\right)$ - банахово пространство гладких функций на $\mathbb{R}^{d}$ с конечной нормой

$$
\|f\|_{N, B}=\sup _{x, \kappa}(1+|x|)^{N} \frac{\left|\partial^{\kappa} f(x)\right|}{B^{|\kappa|} \kappa^{\beta \kappa}} .
$$

(Здесь и далее используются стандартные мультииндексные обозначения, принятые в [23], [28].) Фурье-преобразованное пространство $\widehat{\mathscr{S}}^{\beta}=\mathscr{S}_{\beta}$ относится к классу пространств $K\left(M_{n}\right)$, где в данном случае $M_{n}(p)=e^{n|p|^{1 / \beta}}$, и оно удовлетворяет условию ядерности $\int\left(M_{n} / M_{n^{\prime}}\right) d p<\infty, n^{\prime}>n$, указанному в [23]. Если $\beta>1$, пространство $\mathscr{S}^{\beta}$ содержит функции с компактным носителем, и элементы сопряженного ему пространства $\left(\mathscr{S}^{\beta}\right)^{\prime}$ принято называть ультрараспределениями (типа Бёрлинга) умеренного роста. При $\beta \leqslant 1$ функции из $\mathscr{S}^{\beta}\left(\mathbb{R}^{d}\right)$ аналитически продолжаются на $\mathbb{C}^{d}$ как целые функции порядка $\leqslant 1 /(1-\beta)$. Особую роль играет пространство $\mathscr{S}^{1}$. Вслед за Моримото [35] элементы $\left(\mathscr{S}^{1}\right)^{\prime}$ называют ультрагиперфункциями умеренного роста, они использовались при аксиоматическом построении нелокальной квантовой теории поля с фундаментальной длиной (см. [29], [36], [37] и ссылки в этих статьях). Легко видеть, что для любой вещественной билинейной формы $\theta$ на $\mathbb{R}^{d}$ функция $e^{-i \theta / 2}$ является поточечным мультипликатором пространства $\mathscr{S}_{\beta}\left(\mathbb{R}^{2 d}\right)$. Тем самым для $\mathscr{S}_{\beta}$ выполнены все условия теоремы 1 и $\mathscr{S}^{\beta}$ является алгеброй относительно звездочного произведения $\star_{\theta}$ при любом $\beta>0$. При изучении моделей некоммутативной квантовой теории поля обычно используется представление мойаловского произведения в форме степенного разложения

$$
\left(f \star_{\theta} g\right)(x)=f(x) g(x)+\sum_{n=1}^{\infty}\left(\frac{i}{2}\right)^{n} \frac{1}{n !} \vartheta^{\mu_{1} \nu_{1}} \ldots \vartheta^{\mu_{n} \nu_{n}} \partial_{\mu_{1}} \ldots \partial_{\mu_{n}} f(x) \partial_{\nu_{1}} \ldots \partial_{\nu_{n}} g(x)
$$

где $\vartheta^{\mu \nu}$ - матрица симплектической формы $\theta$. В работе [21] показано, что если $\beta \leqslant 1 / 2$, то ряд (26) абсолютно сходится для всех $f, g \in \mathscr{S}^{\beta}$ по каждой из норм (25). Пространство $\mathscr{S}^{1 / 2}$ является максимальным $\mathrm{FN}$-подпространством в $S$, для которого справедливо представление (26) с абсолютной сходимостью, поэтому оно играет выделенную роль в некоммутативной теории поля. Обозначим через $E_{N}^{\beta, B}\left(\mathbb{R}^{d}\right)$ банахово пространство гладких функций на $\mathbb{R}^{d}$ с конечной нормой

$$
\|f\|_{-N, B}=\sup _{x, \kappa}(1+|x|)^{-N} \frac{\left|\partial^{\kappa} f(x)\right|}{B^{|\kappa|} \kappa^{\beta \kappa}}
$$

и пусть $\mathscr{E}^{\beta}=$ inj $\lim _{N \rightarrow \infty} \operatorname{proj} \lim _{B \rightarrow 0} E_{N}^{\beta, B}$.

Теорема 4. Пространство $\mathscr{E}^{\beta}$ содержится в $\mathcal{M}_{\theta}\left(\mathscr{S}^{\beta}\right)$. При любых $w \in \mathscr{E}^{\beta}$ $u v \in\left(\mathscr{S}^{\beta}\right)^{\prime}$ мойаловские произведения $w \star_{\theta} v, v \star_{\theta} w$ корректно определены как элементы $\left(\mathscr{S}^{\beta}\right)^{\prime}$.

ДокАЗАтЕльство. Согласно следствию 2 для доказательства первого утверждения достаточно доказать включение $\mathscr{E}^{\beta} \subset C^{\prime}\left(\mathscr{S}^{\beta}\right)$. Тогда верно и второе утверждение, поскольку указанные произведения определены по дуальности для любых 
$w \in \mathcal{M}_{\theta}\left(\mathscr{S}^{\beta}\right), v \in\left(\mathscr{S}^{\beta}\right)^{\prime}$. В работе [24] доказано ${ }^{4)}$, что алгебра $C\left(\mathscr{S}^{\beta}\right)$ состоит из тех же элементов, что и пространство $\left(\mathscr{E}^{\beta}\right)^{\prime}$. Мы покажем, что топология $C\left(\mathscr{S}^{\beta}\right)$ не слабее, чем $\sigma\left(\left(\mathscr{E}^{\beta}\right)^{\prime}, \mathscr{E}^{\beta}\right)$, и, следовательно, $C^{\prime}\left(\mathscr{S}^{\beta}\right) \supset\left(\left(\mathscr{E}^{\beta}\right)_{\sigma}^{\prime}\right)^{\prime}=\mathscr{E}^{\beta}$.

Если функционал $u \in\left(\mathscr{S}^{\beta}\right)^{\prime}$ принадлежит $C\left(\mathscr{S}^{\beta}\right)$, то его непрерывное продолжение на $\mathscr{E}^{\beta}$ можно построить следующим образом. Пусть $f_{0}-$ функция в $\mathscr{S}^{\beta}$ со свойством $\int f_{0}(\xi) d \xi=1$ и пусть $h \in \mathscr{E}^{\beta}$. Положим

$$
\langle u, h\rangle=\int\left\langle u, h(\cdot) f_{0}(\xi-\cdot)\right\rangle d \xi=\int\left(u * h_{\xi}\right)(\xi) d \xi, \quad h_{\xi}(x) \stackrel{\text { def }}{=} h(\xi-x) f_{0}(x)
$$

(здесь звездочка * обозначает обычную свертку). Подынтегральная функция в (28) непрерывна, поскольку сдвиги действуют в $\mathscr{S}^{\beta}$ непрерывно, а $h$ является поточечным мультипликатором для этого пространства. Существует $N_{0}$ такое, что $\|h\|_{-N_{0}, B}<\infty$ при любом $B>0$, поэтому для функции $h_{\xi} \in \mathscr{S}^{\beta}$ справедлива оценка

$$
\begin{aligned}
\left|\partial^{\kappa} h_{\xi}(x)\right| \leqslant & \sum_{\mu}\left(\begin{array}{c}
\kappa \\
\mu
\end{array}\right)\left|\partial^{\mu} h(\xi-x) \partial^{\kappa-\mu} f_{0}(x)\right| \leqslant \\
\leqslant & \|h\|_{-N_{0}, B}\left\|f_{0}\right\|_{N, B} \sum_{\mu}\left(\begin{array}{c}
\kappa \\
\mu
\end{array}\right) B^{|\mu|} \mu^{\beta \mu} B^{|\kappa-\mu|}(\kappa-\mu)^{\beta(\kappa-\mu)} \times \\
& \times(1+|\xi-x|)^{N_{0}}(1+|x|)^{-N} \leqslant \\
\leqslant & \|h\|_{-N_{0}, B}\left\|f_{0}\right\|_{N, B}(2 B)^{|\kappa|} \kappa^{\beta \kappa}(1+|x|)^{N_{0}-N}(1+|\xi|)^{N_{0}} .
\end{aligned}
$$

Отсюда получаем, что при любых $N, B>0$

$$
\left\|h_{\xi}\right\|_{N, B} \leqslant\|h\|_{-N_{0}, B / 2}\left\|f_{0}\right\|_{N+N_{0}, B / 2}(1+|\xi|)^{N_{0}} .
$$

Поскольку $u \in C\left(\mathscr{S}^{\beta}\right)$, для окрестности $U=\left\{g:\|g\|_{N_{0}+d+1,1}<1\right\}$ в $\mathscr{S}^{\beta}$ найдется окрестность $V=\left\{g:\|g\|_{N, B} \leqslant \delta\right\}$ такая, что $u * g \in U$ при всех $g \in V$. Обозначим правую часть неравенства (30) через $\lambda_{\xi}$. Тогда $\delta \lambda_{\xi}^{-1} h_{\xi} \in V$ и, следовательно,

$$
\left|\left(u * h_{\xi}\right)(\xi)\right| \leqslant \delta^{-1} \lambda_{\xi}(1+|\xi|)^{-N_{0}-d-1}=\delta^{-1}\|h\|_{-N_{0}, B / 2}\left\|f_{0}\right\|_{N+N_{0}, B / 2}(1+|\xi|)^{-d-1} .
$$

Таким образом, интеграл в (28) абсолютно сходится и функционал $u$ корректно определен на $\mathscr{E} \beta$. Поскольку правая часть (31) содержит фактор $\|h\|_{-N_{0}, B / 2}$, этот функционал непрерывен на каждом пространстве $\operatorname{proj} \lim _{B \rightarrow 0} \mathscr{E}_{N}^{\beta, B}$, а тем самым и на $\mathscr{E}^{\beta}$. Надо еще убедиться, что на пространстве $\mathscr{S}^{\beta}$ определяемый формулой (28) функционал совпадает с исходным, что оправдывает использование для него того же обозначения. Если $h \in \mathscr{S}^{\beta}$, то аналогичная (29) оценка показывает, что при любых $N_{0}, N$ и $B$ справедливо неравенство

$$
\left\|h(x) f_{0}(\xi-x)\right\|_{N, B} \leqslant\|h\|_{N+N_{0}, B / 2}\left\|f_{0}\right\|_{N_{0}, B / 2}(1+|\xi|)^{-N_{0}} .
$$

Отсюда следует, что интеграл в (28) остается абсолютно сходящимся при замене $u$ на любой функционал $v \in\left(\mathscr{S}^{\beta}\right)^{\prime}$, ибо $\|v\|_{N, B} \leqslant \infty$ при некоторых $N$ и $B$. Значит, соответствующая последовательность интегральных сумм слабо фундаментальна в $\mathscr{S}^{\beta}$.

4) В [24] вместо $\mathscr{S}^{\beta}$ и $\mathscr{E}^{\beta}$ использовались обозначения $S^{\beta-}$ и $\widehat{\mathscr{E}} \beta-$. Там показано, что пространством поточечных мультипликаторов для $\mathscr{S}^{\beta}$ является proj $\lim _{B \rightarrow 0} \operatorname{inj} \lim _{N \rightarrow \infty} E_{N}^{\beta, B}$. 
Поскольку пространство $\mathscr{S}^{\beta}$ монтелевское, оно полно относительно слабой сходимости и в нем слабая сходимость влечет сильную (см. [23], § I.6.3). Следовательно, последовательность интегральных сумм сходится в $\mathscr{S}^{\beta}$, а ее пределом может быть только $h$, так как топология $\mathscr{S}^{\beta}$ сильнее топологии простой сходимости.

Пусть теперь $u_{\gamma} \rightarrow 0$ в $C\left(\mathscr{S}^{\beta}\right)$. Тогда для ограниченного множества

$$
Q=\bigcap_{N, B}\left\{g:\|g\|_{N, B} \leqslant\|h\|_{-N_{0}, B / 2}\left\|f_{0}\right\|_{N+N_{0}, B / 2}\right\}
$$

и окрестности $\epsilon U=\left\{g:\|g\|_{N_{0}+d+1,1}<\epsilon\right\}$ со сколь угодно малым $\epsilon$ найдется такое $\gamma_{0}$, что $u_{\gamma} * g \in \epsilon U$ при всех $g \in Q$ и $\gamma>\gamma_{0}$. Согласно (30) семейство функций $(1+|\xi|)^{-N_{0}} h_{\xi}$ содержится в $Q$, и мы заключаем, что

$$
\left|\left\langle u_{\gamma}, h\right\rangle\right| \leqslant \int\left|\left(u_{\gamma} * h_{\xi}\right)(\xi)\right| d \xi \leqslant \epsilon \int(1+|\xi|)^{-d-1} d \xi \quad \text { при всех } \gamma>\gamma_{0} .
$$

Следовательно, $\left\langle u_{\gamma}, h\right\rangle \rightarrow 0$ при любом $h \in \mathscr{E}^{\beta}$, что завершает доказательство.

Пространства Гельфанда-Шилова $S_{\alpha}^{\beta}$ являются пространствами DFN, алгебры $\mathcal{M}_{\theta}\left(S_{\alpha}^{\beta}\right)$ построены и изучены в работе [25]. Там же показана выделенная роль минимального нетривиального инвариантного относительно преобразования Фурье пространства $S_{1 / 2}^{1 / 2}$. Другие часто используемые пространства Гельфанда-Шилова $S^{\beta}=\operatorname{inj} \lim _{B \rightarrow \infty} \operatorname{proj} \lim _{N \rightarrow \infty} S_{N}^{\beta, B}$ не являются ни пространствами Фреше, ни пространствами DF. В эту шкалу входит пространство $S^{0}$, представляющее собой фурье-образ пространства $\mathscr{D}$ гладких функций с компактным носителем. Возможность использования пространства $S^{0}$ в некоммутативной теории поля обсуждалась в работах [17], [38]. Следует отметить, что пространства $S^{\beta}$ с $\beta>0$ не наделялись топологией в [23], где вместо этого использовалось понятие сходимости последовательностей. Тем не менее к ним применимо вышеизложенное рассмотрение, поскольку при снабжении естественной топологией они ядерны [39], полны [40] и, очевидно, бочечны как индуктивные пределы пространств Фреше. В работе [24] доказано, что алгебра $C\left(S^{\beta}\right)$ состоит из тех же элементов, что и пространство, сопряженное к $E^{\beta}=\operatorname{inj} \lim _{N, B \rightarrow \infty} E_{N}^{\beta, B}=M\left(S^{\beta}\right)$.

Теорема 5. Пространство $E^{\beta}$ содержится в $\mathcal{M}_{\theta}\left(S^{\beta}\right)$. При любъх $w \in E^{\beta} u$ $v \in\left(S^{\beta}\right)^{\prime}$ мойаловские произведения $w \star_{\theta} v, v \star_{\theta} w$ корректно определены как элементы $\left(S^{\beta}\right)^{\prime}$.

ДокАзАтельство. Аналогично разобранному выше случаю $\mathscr{S}^{\beta}$, достаточно показать, что топология пространства $C\left(S^{\beta}\right)$ не слабее, чем $\sigma\left(\left(E^{\beta}\right)^{\prime}, E^{\beta}\right)$. Непрерывное продолжение функционала $u \in\left(S^{\beta}\right)^{\prime}$ на $E^{\beta}$ можно определить той же формулой $(28)$ с $f_{0} \in S^{\beta}$. На этот раз $\|h\|_{-N_{0}, B_{0}}<\infty$ при некоторых $N_{0}, B_{0}>0$, и аналогичная (29) оценка дает

$$
\left\|h_{\xi}\right\|_{N, B_{0}} \leqslant\|h\|_{-N_{0}, B_{0} / 2}\left\|f_{0}\right\|_{N+N_{0}, B_{0} / 2}(1+|\xi|)^{N_{0}} \quad \text { при любом } N>0 .
$$

Значит, семейство функций $(1+|\xi|)^{-N_{0}} h_{\xi}$ ограничено в $S^{\beta}$.

Пусть $U$ - окрестность нуля в $S^{\beta}$, определяемая как абсолютно выпуклая оболочка множества $\cup_{B}\left\{g:\|g\|_{N_{0}+d+1, B}<1\right\}$. Тогда все функции в $U$ мажорируются 
функцией $(1+|x|)^{-N_{0}-d-1}$. Поскольку образ любого ограниченного множества при непрерывном отображении $g \rightarrow u * g$ поглощается окрестностью $U$, мы заключаем, что интеграл в (28) сходится и определяет линейный непрерывный функционал на $E^{\beta}$. Если $h \in S^{\beta}$, то в данном случае неравенство (32) соблюдается при некотором $B$ и любых $N, N_{0}$. Значит, интеграл в (28) абсолютно сходится при замене $u$ на любой линейный функционал, определенный и непрерывный на монтелевском пространстве proj $\lim _{N \rightarrow \infty, \varepsilon \rightarrow 0} S_{N}^{\beta, B_{1}+\varepsilon}$, где $B_{1}>B$, и мы заключаем, что на $S^{\beta}$ построенный функционал совпадает с исходным. Наконец, пусть $u_{\gamma} \rightarrow 0$ в $C\left(S^{\beta}\right)$. Тогда для ограниченного множества

$$
Q=\bigcap_{N}\left\{g:\|g\|_{N, B_{0}} \leqslant\|h\|_{-N_{0}, B_{0} / 2}\left\|f_{0}\right\|_{N+N_{0}, B_{0} / 2}\right\}
$$

и окрестности $\epsilon U$ найдется такое $\gamma_{0}$, что $u_{\gamma} * g \in \epsilon U$ при всех $g \in Q$ и $\gamma>\gamma_{0}$. Согласно (34) семейство $(1+|\xi|)^{-N_{0}} h_{\xi}$ содержится в $Q$, и мы приходим к (33), что завершает доказательство.

Мы видим, что алгебры $\mathcal{M}_{\theta}\left(\mathscr{S}^{\beta}\right)$ и $\mathcal{M}_{\theta}\left(S^{\beta}\right)$ при любом $\theta$ включают в себя все полиномы, как и алгебра мойаловских мультипликаторов для пространства Шварца. Кроме того, при любом $\beta \geqslant \beta_{0}, 0<\beta_{0}<1$, эти алгебры содержат целые функции порядка роста не больше $1 /\left(1-\beta_{0}\right)$ (в случае $\mathcal{M}_{\theta}\left(\mathscr{S}^{\beta_{0}}\right)$ минимального типа), которые полиномиально ограничены на вещественном пространстве. В частности, при любом $\beta \geqslant 0$ алгебра $\mathcal{M}_{\theta}\left(S^{\beta}\right)$ содержит пространство $\mathscr{O}_{\text {exp }}$, состоящее из целых функций экспоненциального типа, удовлетворяющих оценке $|f(z)| \leqslant C(1+|z|)^{N} e^{b|\operatorname{Im} z|}$, где константы $C, N$ и $b$ зависят от $f$. Это обобщает результат [8], [9], установленный ранее для $\mathcal{M}_{\theta}(S)$.

ЗАмЕЧАниЕ 4. Используя рассуждение, аналогичное доказательству леммы 1 в работе [25] для $S_{\alpha}^{\beta}$, можно показать, что $\mathscr{S}^{\beta}$ плотно в $\mathcal{M}_{\theta}\left(\mathscr{S}^{\beta}\right)$. В соответствии со сказанным в конце раздела 3 отсюда следует, что $\mathcal{M}_{\theta}\left(\mathscr{S}^{\beta}\right)$ является алгеброй относительно мойаловского произведения, а $\left(\mathscr{S}^{\beta}\right)^{\prime}$ является $\mathcal{M}_{\theta}\left(\mathscr{S}^{\beta}\right)$-бимодулем. Аналогичным образом, $\left(S^{\beta}\right)^{\prime}$ есть бимодуль над алгеброй $\mathcal{M}_{\theta}\left(S^{\beta}\right)$.

Вопрос о преобразовании Вейля построенных алгебр мойаловских мультипликаторов, т. е. об их операторной реализации в гильбертовом пространстве, будет рассмотрен в последующей статье.

\section{ПРИЛОЖЕНИЕ}

При рассмотрении линейных представлений топологических групп в монтелевских функциональных пространствах полезна следующая простая лемма.

ЛЕмма 1. Предположим, что $E$ - монтелевское пространство функиий на множестве $X$ и топология Е сильнее топологии поточечной сходимости. Пусть в $E$ задано линейное представление $\mathcal{T}$ локально компактной группы $\mathcal{G}$. Если для каждой функиии $f \in E$ существует компактная окрестность $\mathcal{K}$ единиць е группљ $\mathcal{G}$ такал, что множество $\left\{\mathcal{T}_{a} f: a \in \mathcal{K}\right\}$ ограничено в $E$, и если $\lim _{a \rightarrow e}\left(\mathcal{T}_{a} f\right)(x)=$ $f(x)$ при всех $x \in X$, то представление $\mathcal{T}$ непрерывно. 
ДоКАЗАТЕЛьство. Если $\left\{a_{n}\right\}$ - последовательность, сходящаяся к $e$ в $\mathcal{G}$, то $a_{n} \in \mathcal{K}$ при достаточно больших $n$ и, значит, последовательность $\left\{\mathcal{T}_{a_{n}} f\right\}$ ограничена в $E$. По определению монтелевского пространства [26] такая последовательность имеет по крайней мере одну точку прикосновения. Поскольку топология $E$ сильнее топологии простой сходимости, из предельного соотношения $\lim _{a \rightarrow e}\left(\mathcal{T}_{a} f\right)(x)=f(x)$ следует, что точкой прикосновения может быть только $f$, и тем самым $\mathcal{T}_{a_{n}} f \rightarrow f$. Далее, к монтелевским пространствам применима обобщенная теорема БанахаШтейнгауза ([26], § III.4.2), согласно которой из поточечной ограниченности множества операторов $\left\{\mathcal{T}_{a}\right\}_{a \in \mathcal{K}}$ следует, что для всякой абсолютно выпуклой окрестности нуля $U$ в $E$ найдется окрестность $V$ такая, что $\mathcal{T}_{a}(V) \subset U$ при всех $a \in \mathcal{K}$. Записывая $\mathcal{T}_{a} f-f_{0}=\mathcal{T}_{a}\left(f-f_{0}\right)+\mathcal{T}_{a} f_{0}-f_{0}$, мы видим, что для окрестности $U$ и всякой функции $f_{0} \in E$ найдется окрестность $\mathcal{K}^{\prime}(e)$ такая, что $\mathcal{T}_{a} f \in f_{0}+U$ при всех $f \in f_{0}+V / 2$ и $a \in \mathcal{K}^{\prime}$, т. е. отображение $\mathcal{G} \times E \rightarrow E:(a, f) \rightarrow \mathcal{T}_{a} f$ непрерывно в точке $\left(e, f_{0}\right)$. Записывая $\mathcal{T}_{a} f-\mathcal{T}_{a_{0}} f_{0}=\mathcal{T}_{a_{0}}\left(\mathcal{T}_{a_{0}^{-1}} f-f_{0}\right)$, мы заключаем, что это отображение непрерывно всюду. Лемма доказана.

Благодарности. Работа поддержана РФФИ (грант № 09-01-00835).

\section{Список литературы}

[1] Ф.А. Березин, М. А. Шубин, Уравнение Шредингера, Изд-во МГУ, М., 1983.

[2] C. K. Zachos, D. B. Fairlie, T. L. Curtright (eds.), Quantum Mechanics in Phase Space, World Scientific Series in 20th Century Physics, 34, World Scientific, Singapore, 2005.

[3] J. von Neumann, Math. Ann., 104:1 (1931), 570-578.

[4] M. A. Antonets, Lett. Math. Phys., 2:3 (1978), 241-245.

[5] М. А. Антонец, Матем. сб., 107(149):1(9) (1978), 20-36.

[6] М. А. Антонец, ТМФ, 38:3 (1979), 331-344.

[7] J. M. Maillard, J. Geom. Phys., 3:2 (1986), 231-261.

[8] J. M. Gracia-Bondia, J. C. Várilly, J. Math. Phys., 29:4 (1988), 869-879; J. C. Várilly, J. M. Gracia-Bondia, J. Math. Phys., 29:4 (1988), 880-887.

[9] R. Estrada, J. M. Gracia-Bondia, J. C. Várilly, J. Math. Phys., 30:12 (1989), 2789-2796.

[10] V. Gayral, J. M. Gracia-Bondia, B. Iochum, T. Schücker, J. C. Várilly, Commun. Math. Phys., 246:3 (2004), 569-623, arXiv: hep-th/0307241.

[11] R. J. Szabo, Phys. Rep., 378:4 (2003), 207-299, arXiv: hep-th/0109162.

[12] S. Doplicher, K. Fredenhagen, J. E. Roberts, Phys. Lett. B, 331:1-2 (1994), 39-44.

[13] S. Doplicher, K. Fredenhagen, J. E. Roberts, Commun. Math. Phys., 172:1 (1995), 187-220, arXiv: hep-th/0303037.

[14] N. Seiberg, E. Witten, JHEP, 09 (1999), 032, 93 pp., arXiv: hep-th/9908142.

[15] L. Álvarez-Gaumé, M. A. Vázquez-Mozo, Nucl. Phys. B, 668:1-2 (2003), 293-321, arXiv: hep-th/0305093.

[16] V.E. Hubeny, M. Rangamani, S.F. Ross, JHEP, 07 (2005), 037, 32 pp., arXiv: hep-th/0504034.

[17] М. А. Соловьев, ТМФ, 147:2 (2006), 257-269, arXiv: hep-th/0605249.

[18] М. А. Соловьев, ТМФ, 153:1 (2007), 3-17, arXiv: 0708.0811.

[19] M. Chaichian, M. Mnatsakanova, A. Tureanu, Yu. Vernov, JHEP, 09 (2008), 125, 11 pp., arXiv: 0706.1712.

[20] O. W. Greenberg, Phys. Rev. D, 73:4 (2006), 045014, 5 pp., arXiv: hep-th/0508057.

[21] M. A. Soloviev, J. Phys A, 40:48 (2007), 14593-14604, arXiv: 0708.1151.

[22] M. A. Soloviev, Phys. Rev. D, 77:12 (2008), 125013, 11 pp., arXiv: 0802.0997. 
[23] И. М. Гельфанд, Г. Е. Шилов, Обобщенные функиии, Вып. 2: Пространства основных и обобщенных функиий, Физматгиз, М., 1958.

[24] В. П. Паламодов, Труды МMO, 11 (1962), 309-350.

[25] M. A. Soloviev, J. Math. Phys., 52:6 (2011), 063502, 18 pp., arXiv: 1012.0669.

[26] Х. Шефер, Топологические векторные пространства, Мир, М., 1966.

[27] В. И. Арнольд, Математические методы классической механики, Наука, М., 1989.

[28] Л. Хёрмандер, Анализ линейных дифференциальных операторов с частными производными, т. 1: Теория распределений и анализ Фурье, Мир, М., 1986.

[29] M. A. Soloviev, J. Math. Phys., 51:9 (2010), 093520, 20 pp., arXiv: 1012.3546.

[30] A. Grothendieck, Mem. Amer. Math. Soc., 16 (1955), 140 pp.

[31] G. Köthe, Topological Vector Spaces. II, Grundlehren der Mathematischen Wissenschaften [Fundamental Principles of Mathematical Science], 237, Springer, New York, 1979.

[32] В. В. Жаринов, УМН, 34:4(208) (1979), 97-131.

[33] M. Morimoto, An Introduction to Sato's Hyperfunctions, Translations of Mathematical Monographs, 129, AMS, Providence, RI, 1993.

[34] М. А. Соловьев, ТМФ, 163:3 (2010), 413-429, arXiv: 1012.3536.

[35] M. Morimoto, Proc. Japan Acad., 51:2 (1975), 87-91; 51:4 (1975), 213-218.

[36] E. Brüning, S. Nagamachi, J. Math. Phys., 45:6 (2004), 2199-2231.

[37] M. A. Soloviev, J. Math. Phys., 50:12 (2009), 123519, 17 pp., arXiv: 0912.0595.

[38] D. H. T. Franco, C. M. M. Polito, J. Math. Phys., 46:8 (2005), 083503, 11 pp., arXiv: hep-th/0403028.

[39] Б. С. Митягин, Трудъ ММО, 9 (1960), 317-328.

[40] М. А. Соловьев, ТМФ, 128:3 (2001), 492-514, arXiv: math-ph/0112052.

Поступила в редакцию 12.09.2011 